\title{
Lineamientos de un sistema integrado de gestión bajo los requisitos de NTC-ISO 9001:2015 y NTC-ISO 14001:2015 en instituciones de educación superior ${ }^{*}$
}

Guidelines of an integrated management system under the requirements of NTC-ISO 9001:2015 and NTC-ISO 14001:2015 in institutions of superior education

Diretrizes de um sistema de gerenciamento integrado de acordo com os requisitos das NTC ISO 9001: 2015 e NTC ISO 14001:

2015 em instituições de ensino superior

Sandra Jastri Losada Rodríguez***

Universidad Santo Tomás

Sonia Angelica Zambrano Pineda***

Universidad Nacional de Colombia

* Artículo de resultado de investigación. DOI: http://dx.doi.org/10.15332/s2145-1389.2017.0001.05

** Magíster (c) en Calidad y Gestión Integral. Especialista en Administración y Gerencia de Sistemas de la Calidad. Ingeniera Logística. Profesional especializada Unidad de Gestión Integral de la Calidad Universitaria de la Universidad Santo Tomás. Correo electrónico: sandralosada@hotmail. com, sandralosada@usantotomas.edu.co.

*** Magíster (c) en Calidad y Gestión Integral. Especialista en Administración y Gerencia de Sistemas de la Calidad. Microbióloga Industrial. Profesional en Calidad de la Universidad Nacional de Colombia. Correo electrónico: sonyaangel25@yahoo.es. 


\section{RESUMEN}

Con el proposito de realizar una propuesta de lineamientos para integrar los sistemas de gestión según las normas técnicas NTC-ISO 9001:2015 y NTC-ISO 14001:2015 en instituciones de educación superior (IES), se tomó una muestra de seis universidades certificadas por Icontec con la norma técnica NTC-ISO 9001, en las cuales se realizó un análisis de sus documentos maestros para identificar ejes y componentes misionales que puedan facilitar la articulación de un sistema integrado de gestión. Posteriormente se realizó un diagnóstico para identificar el estado actual frente a la gestión ambiental y a la integración de dichas normas. Los resultados muestran que el sistema de calidad está implícito desde la planeación estratégica, hace parte de su cultura organizacional y es una herramienta que contribuye al cumplimiento de la misión. Con la gestión ambiental no se refleja esta misma apropiación al evidenciarse ausencia de elementos como políticas o lineamientos que contribuyan a identificar, disminuir 0 controlar los aspectos ambientales. Finalmente se propusieron unos lineamientos que pueden servir para que las IES integren sus sistemas de gestión de calidad y ambiental, de manera que aporten a la toma de decisiones por parte de la alta dirección.

Palabras clave: instituciones de educación superior, sistema de gestión ambiental, sistema de gestión de la calidad, sistema integrado de gestión.

\section{ABSTRACT}

In order to make a proposal for guidelines to facilitate the integration of systems under NTC-ISO 9001:2015 and NTC-ISO 14001:2015 in higher education institutions (HEI), a sample of six universities certified with Icontec in NTC-ISO 9001, in which an analysis of its master documents was made to identify axes and missionary components that allow the articulation of an integrated management system. Subsequently a diagnosis was made to identify the current state of the environmental management and the integration of these standards. The results show that the quality system is implicit from the strategic planning, is part of its organizational culture and is a tool that contributes to the fulfillment of the mission. With environmental management, this same appropriation is not reflected in the absence of elements such as policies or guidelines that contribute to identify, reduce or control aspects. Finally, with these elements, guidelines were proposed that serve as a tool for the HEI to integrate their quality and environmental management systems and to contribute to the decision-making by top management.

Keywords: Higher education institutions, environmental management system, quality management system, integrated management system.

\section{RESUMO}

Com o propósito de realizar uma proposta de diretrizes que facilitem a integração dos sistemas sob a NTC ISO 9001: 2015 e NTC ISO14001: 2015, nas instituições de ensino superior, foram tomadas amostra de seis universidades certificadas com ICONTEC com a NTC ISO 9001, nas quais se realizou uma análise dos seus documentos mestre para identificar eixos e componentes missionários que permitam a articulação de um sistema de gestão integrada. Posteriormente, realizou-se um diagnóstico para identificar o estado atual em relação a gestão ambiental e a integração dessas normas.

Os resultados mostram que 0 sistema de qualidade está implícito desde o planejamento estratégico, faz parte da sua cultura organizacional e é uma ferramenta 
que contribui para ao cumprimento da missão. Com a gestão ambiental não se reflete esta mesma apropriação por ausência de elementos, como políticas ou diretrizes que ajudem a identificar, reduzir ou controlar aspectos.

Finalmente com esses elementos, foram propostas algumas diretrizes que servem como ferramenta para que as IES integrem seus sistemas de gestão da qualidade e ambiental e contribuir para a tomada de decisões da alta diretoria.

Palavras-chave: estrutura de alto nível, instituições de ensino superior, sistema de gestão ambiental, sistema de gestão da qualidade, sistema integrado de gestão.

\section{INTRODUCCIÓN}

Este artículo busca que las instituciones de educación superior en su interés de aumentar el logro de sus propósitos y satisfacer las necesidades de sus usuarios y de sus partes interesadas y que han optado por implementar un sistema de gestión de la calidad (SGC), siguiendo el modelo NTC-ISO 9001, cuenten con argumentos ecológicos y sociales en su gestión integral, desde una perspectiva sistémica del ambiente en sus dimensiones natural, social y económica. Es decir, "enfrentar probablemente el más complejo reto que los negocios han enfrentado, que consiste en organizarse de manera armónica con los sistemas naturales y sociales, y asegurar prosperidad a corto y a largo plazo" (Ramírez y Manrique, 2012).

Es importante tener claro que las IES realizan diversas actividades con implicaciones ambientales de extensa condición. Dichas implicaciones van desde la generación de residuos, incluidos aquellos peligrosos producidos en centros médicos y laboratorios, hasta altos consumos de recursos como energía y agua con graves consecuencias sobre su entorno (Rivas, 2011). Los aspectos positivos podrían ser desarrollados para generar conciencia en los nuevos profesionales sobre la importancia del medio ambiente, la iniciativa de las instituciones en proponer y fomentar cambios en los programas educativos, destacando particularmente programas académicos relacionados con el desarrollo sostenible en el mundo y las estrategias recomendadas para garantizar una educación de calidad para la sostenibilidad, donde el tema ambiental ejerce una influencia positiva y definitiva en la mejora de la calidad de vida (Ferguson, García y Bornay, 2002).

De acuerdo con lo anterior, el presente estudio tiene como objetivo central proponer lineamientos para la integración de sistema de gestión NTC-ISO 9001 y NTC-ISO 14001 en las IES. El estudio se lleva a cabo con una muestra de seis universidades que se encuentran certificadas con Icontec bajo la NTC-ISO 9001:2008. Para lograr esto se realiza un estudio cualitativo con diseño fenomenológico, mediante el análisis de los documentos institucionales con el fin de identificar los ejes y componentes misionales para articular en ellos los componentes de un sistema de gestión; se realiza un diagnóstico a partir de entrevistas semiestructuradas para identificar el estado actual de las IES frente a la gestión ambiental y a la integración de las normas. Con los resultados se elabora la propuesta de los lineamientos, que se somete a una validación de contenido por parte de jueces, los cuales fueron seleccionados por su experiencia en sistemas de gestión de calidad y ambiental.

Este estudio podrá ser utilizado en futuras investigaciones con el fin de ser aplicado en IES que deseen contar con un sistema integrado de gestión bajo los lineamientos de la NTC-ISO 9001:2015 y NTC-ISO 14001:2015; asimismo pretende convertirse en una herramienta que contribuya al desarrollo de las IES 
permitiéndoles ser cada día más competentes, como por ejemplo contribuir a la acreditación de alta calidad, ampliar las oportunidades para su internacionalización, fortalecer los canales de comunicación, facilitar los procesos de los ejercicios evaluativos (auditorías internas y externas), toma de decisiones de manera integrada y abordar el tema ambiental más allá de los muros universitarios.

De acuerdo con los niveles de integración de sistemas de gestión presentados por autores como Wilkinson y Dale (1999), Bernardo et.al. (2009) y Karapetrovic (2003), es posible establecer una cierta coincidencia; por ejemplo, en el primer nivel de integración cada norma/sistema se maneja separadamente y el tercer nivel se evidencia cuando los sistemas concretos se integran en un sistema de gestión total, donde las políticas y los objetivos están alineados con la estrategia de la organización. Se resalta lo planteado por el autor Karapetrovic, donde el propósito de un sistema integrado de gestión es brindar una estructura para una administración total, que integre los aspectos comunes de los distintos subsistemas para evitar duplicaciones, y de esta manera, mejorar la eficacia y eficiencia de la organización (Karapetrovic, 2003).

\section{METODOLOGÍA}

La investigación se desarrolla desde el paradigma epistemológico positivista que busca, a partir de la observación y la aplicación de instrumentos cualitativos, establecer las relaciones causa-efecto, con el fin de generar lineamientos para el sistema integrado de gestión bajo los lineamientos de las normas mencionadas.

El diseño de esta investigación corresponde a un diseño fenomenológico, el cual explora, describe y comprende las experiencias de las personas con respecto a un fenómeno, en este caso las normas NTC-ISO 9001:2015 y NTC-ISO 14001:2015.

Con respecto a los tipos tradicionales de investigación, el presente estudio se clasifica como una investigación exploratoria, teniendo en cuenta que la revisión bibliográfica inicial realizada para definir el problema a investigar, evidencia que el tema no ha sido abordado a profundidad (Hernández, Fernández y Baptista, 2014).

En cuanto a la población del estudio y realizando un muestreo por conveniencia, se decidió trabajar con una muestra de seis universidades certificadas bajo los lineamientos de la NTC-ISO 9001, así, tres universidades: Universidad Santo Tomás de Aquino, Universidad Sergio Arboleda, Universidad Militar Nueva Granada; tres fundaciones universitarias: Fundación Universitaria los Libertadores, Fundación Universitaria para el Desarrollo Humano - UNINPAHU, Fundación Universitaria de Ciencias de la Salud.

Para el objetivo "identificar los ejes y componentes misionales de las IES para articularlos con un sistema integrado de gestión bajo las NTC-ISO 9001:2015 y NTC-ISO 14001:2015", se llevó a cabo una revisión documental de acuerdo con los siguientes criterios: estudio de documentos institucionales como el Plan Educativo Institucional (PEI), manuales de calidad, documentos marcos y acuerdos de rectorías; revisión en ellos de misión, visión, política, objetivos, alcance y estructura del sistema.

Para el segundo objetivo, "elaborar un diagnóstico de identificación del estado actual en las IES frente a la gestión ambiental y a la integración de las NTCISO 9001:2015 y NTC-ISO 14001:2015”, se aplicó una entrevista semiestructurada. Para determinar las preguntas básicas del instrumento se establecieron unas preguntas germinales, apoyadas en elementos 
conceptuales identificados en el marco teórico; estas preguntas buscaban conocer e identificar el estado de las IES frente a la gestión ambiental y a los sistemas integrados de gestión, así:

1. ¿Qué compromisos y problemáticas internas ambientales se presentan en las IES?

2. ¿Qué se entiende por sistema integrado de gestión?

3. ¿Qué acciones podría realizar la IES para beneficiar los ambientes del entorno concreto?

4. ¿Qué razones tiene la IES para no certificarse en ISO 14001?

Para la sistematización de la información se utilizó el software ATLAS.ti, cuyo fundamento teórico se basa en la teoría de Grounded Theory de Glaser y Strauss y Varguillas. ATLAS.ti es un programa de análisis cualitativo asistido por computadora (QDA), le permite al investigador: a) asociar códigos o etiquetas con fragmentos de texto, sonidos, imágenes, dibujos, videos y otros formatos digitales que no pueden ser analizados significativamente con enfoques formales y estadísticos; b) buscar códigos de patrones; c) clasificarlos ${ }^{1}$.

Posteriormente, para la formulación de los lineamientos para un sistema de gestión integrado bajo las normas NTC-ISO 9001:2015 y NTC-ISO 14001:2015, se realizó un análisis de la información obtenida de la revisión documental, de los resultados del análisis de las entrevistas a través del ATLAS.ti y de la estructura de alto nivel definida en la versión 2015 de las normas NTC-ISO 9001 y NTC-ISO 14001 y algunos elementos propuestos en el libro The integrated use of management system standards (2008).

1 Consultado en internet: http://atlasti.com/2014/06/12/utilizaciondel-programa-de-analisis-cualitativo-atlas-ti-para-gestionar-y-analizar-datos/
Para el objetivo tres, "validar, mediante la técnica de jueces, el contenido de los lineamientos para un sistema de gestión integrado bajo las normas NTC-ISO 9001:2015 y NTC-ISO 14001:2015 en instituciones de educación superior", se utilizó una validación de contenido mediante seis jueces evaluadores a quienes se les solicitó por escrito su apoyo y se les anexó la versión de lineamientos. A continuación se detallan los criterios mediante los cuales los jurados validaron la propuesta de lineamientos:

- Claridad: los conceptos, las ideas y desgloses son lo suficientemente claros.

- Pertinencia: el contenido de cada capítulo de la propuesta realmente da respuesta al capítulo pertinente.

- Suficiencia: el contenido de la propuesta está completo y no necesita de nuevos elementos.

- Relevancia: el contenido de la propuesta es significativo para las IES privadas.

- Aplicación: la propuesta es aplicable en las IES privadas.

\section{RESULTADOS Y DISCUSIÓN}

\section{Elementos institucionales}

Una vez analizados los documentos estratégicos de las IES del estudio, se puede evidenciar que estas plasman en su planeación estratégica los planes de desarrollo institucionales de la vigencia correspondiente.

Como elementos comunes en esta planeación se encuentra el enfoque de la misión con la formación integral de los estudiantes, entendiendo dicho concepto como el proceso mediante el cual los ciudadanos 
son autónomos, idóneos, íntegros, emprendedores y socialmente responsables y responden de manera cívica, ética, creativa y crítica a las exigencias de la vida humana y estén en condiciones de aportar soluciones a las problemáticas y necesidades de la sociedad y del país. Personas con sentido de lo estético y lo político, con actitud investigativa, capaces de adoptar, aplicar y transferir los conocimientos científicos, técnicos y tecnológicos a sus áreas de competencia, a las nuevas condiciones empresariales y a la cambiante realidad del país para contribuir a su desarrollo económico, social y cultural, que promuevan la justicia, la equidad, el respeto por los valores humanos. Esta formación integral es desarrollada por las IES a través del fomento de la investigación, la innovación, la proyección social, la cultura y el emprendimiento.

De igual manera, desde la planeación estratégica de las IES, se observa que estas orientan sus actividades hacia el desarrollo humano, científico, ético y social de sus estudiantes, así mismo buscan desarrollar su capacidad de aprendizaje y de investigación ofreciendo servicios educativos con altos estándares de calidad.

Se puede observar que el objetivo principal siempre es la formación de profesionales e investigadores con una base científica y ética, y con una conciencia crítica, de manera que les permita hacer frente a los requerimientos de un entorno cada vez más globalizado y moderno (Jonker y Karapetrovic, 2004).

Las visiones organizacionales prospectan un futuro, en un escenario a mediano plazo, proyectando tendencias y posibilidades como lo son el liderazgo, la inclusión, la competitividad, la internacionalización y la flexibilidad de sus propuestas educativas para diferentes poblaciones y necesidades de la sociedad. También se observa que buscan satisfacer las necesidades de desarrollo del país a través del fortalecimiento permanente de sus programas académicos.

En lo relacionado con el sistema de gestión de la calidad, las IES de estudio han definido los procesos estratégicos, misionales y de apoyo en donde organizan las actividades de su quehacer diario y que les permite cumplir con los ejes misionales. La política, al igual que la misión, está cohesionada con la formación integral para que los estudiantes respondan a las problemáticas del país. Los alcances de los sistemas de calidad abarcan el diseño y desarrollo de programas académicos y servicios de educación universitaria en programas de pregrado y posgrado, excepto la Universidad Santo Tomás, en donde el alcance solo abarca los programas de pregrado; y la Fundación Universitaria de Ciencias de la Salud, la cual contempla únicamente la gestión de colecciones y servicios de información del sistema de bibliotecas.

Con los elementos nombrados anteriormente, se observa que el sistema de calidad se encuentra implícito desde la planeación estratégica de las IES seleccionadas en la muestra y hace parte de su cultura organizacional, adicionalmente ha sido una herramienta importante que contribuye eficazmente al cumplimiento de la misión.

Sin embargo, en lo relacionado con la gestión ambiental, no se refleja esta misma apropiación, ya que no se menciona ningún elemento ambiental en los componentes estratégicos de las IES. No se observa claramente la definición de políticas o lineamientos que contribuyan a identificar, disminuir o controlar los aspectos ambientales generados por el desarrollo de las actividades propias de las IES. En el análisis de estos documentos no se percibe un compromiso (entendido como la dedicación de recursos tales como tiempo y dinero a la implementación del nuevo sistema) institucional con el medio ambiente. 


\section{Resultados del diagnóstico}

La información analizada en ATLAS.ti fue clasificada en los cinco códigos propuestos inicialmente, no obstante, durante la revisión fue necesario proponer códigos adicionales para obtener un total de 19 códigos de análisis, los cuales fueron modificados en sus nombres de acuerdo con los hallazgos, generalidades y diferencias evidenciadas a lo largo de los documentos.
La mayor densidad de datos se concentró en las categorías relacionadas con la percepción de la norma, así como con las dificultades encontradas para su implementación o para establecer lineamientos que faciliten un proceso de transición hacia la certificación ISO 14001 e integración de los sistemas de calidad y ambiental.

Los 19 códigos se agruparon en seis familias, tal como se ilustra en la tabla 1.

Tabla 1. Familias conformadas de acuerdo a los resultados de ATLAS.ti

\begin{tabular}{|c|c|}
\hline NOMBRE DE LA FAMILIA & DESCRIPCIÓN \\
\hline \multirow{2}{*}{$\begin{array}{l}\text { Familia de información } \\
\text { 1. Sistemas de gestión }\end{array}$} & $\begin{array}{l}\text { Esta aproximación conceptual es la que facilita la comprensión del por qué surgen los sistemas de } \\
\text { gestión y son implementados en las organizaciones y las instituciones de educación superior, ya que } \\
\text { su objetivo primordial consiste en facilitar los procesos de toma de decisiones a través del análisis de } \\
\text { información. Estos resultados brindan la posibilidad de plantear estrategias y acciones orientadas al } \\
\text { cumplimiento de metas e indicadores, para evaluar y verificar cómo se desarrollan los procesos que se } \\
\text { han diseñado como respuesta a necesidades identificadas (Cidad, 2004). }\end{array}$ \\
\hline & $\begin{array}{l}\text { Teniendo identificada la familia de sistemas de gestión, se puede observar que todas las IES del estudio } \\
\text { tienen claridad en cuanto a la definición de sistema de gestión integrado, a la identificación de la } \\
\text { certificación obtenida y a los sistemas de gestión complementarios. De acuerdo a lo anterior, se observa que } \\
\text { para las IES los sistemas de gestión permiten unificar los diferentes procedimientos de una organización, } \\
\text { teniendo en cuenta la gestión de calidad, de seguridad y salud y la gestión ambiental, lo que permite a la } \\
\text { organización la interacción de los procesos a partir de una política, con el fin de poder realizar seguimiento a } \\
\text { la gestión mediante la medición de los objetivos y metas propuestas en la organización. }\end{array}$ \\
\hline \multirow[t]{2}{*}{$\begin{array}{l}\text { Familia de información } \\
\text { 2. Demandas Ambientales }\end{array}$} & $\begin{array}{l}\text { Las IES reconocen que en su gestión como entidades educativas, generan una serie de consecuencias } \\
\text { en diferentes niveles, entre ellos el ambiental; de acuerdo a los diferentes programas de pregrado } \\
\text { o posgrado que ofertan, se encuentran impactos que no solo se ligan a la cantidad de personas que } \\
\text { permanecen en las instalaciones sino a lo que se deriva de las actividades que estos realizan. El consumo } \\
\text { de alimentos, el gasto de elementos como luz y agua, los residuos que se generan de diferentes } \\
\text { tipos, entre otros, son elementos que pueden y deben ser medidos para poder generar indicadores de } \\
\text { desarrollo sostenible que evidencien la importancia de la gestión ambiental y que esta herramienta } \\
\text { puede generar beneficios de gran impacto en el largo plazo. }\end{array}$ \\
\hline & $\begin{array}{l}\text { Los resultados aportan datos sobre problemáticas tales como la falta de políticas de ahorro de agua y } \\
\text { energía, para manejo de residuos sólidos y para la manipulación de alimentos. Sobre las necesidades se } \\
\text { anota la falta de una estrategia para la cultura ambiental y una política de compromiso con los aspectos } \\
\text { ambientales internos y externos. }\end{array}$ \\
\hline $\begin{array}{l}\text { Familia de información } \\
\text { 3. Desarrollo sostenible }\end{array}$ & $\begin{array}{l}\text { Así se comienza a evidenciar que los impactos ambientales son reconocidos por las IES y las personas } \\
\text { encargadas de trabajar en las mismas, mostrando que existe una estrecha relación entre sus actividades, } \\
\text { los objetivos que persiguen y los efectos que las mismas generan, no solo en los niveles de proyección } \\
\text { social, cumplimiento de la misión institucional, entre otros, sino los costos y beneficios sobre los recursos } \\
\text { y el medio ambiente. }\end{array}$ \\
\hline
\end{tabular}




\begin{tabular}{|c|c|}
\hline NOMBRE DE LA FAMILIA & DESCRIPCIÓN \\
\hline $\begin{array}{l}\text { Familia de información } \\
\text { 3. Desarrollo sostenible }\end{array}$ & $\begin{array}{l}\text { El desarrollo sostenible y las líneas de acción orientadas a la búsqueda de su cumplimiento, es } \\
\text { entendido como el "desarrollo que satisface las necesidades de la generación presente, sin comprometer } \\
\text { a las generaciones futuras". Este es un paradigma para pensar en un futuro en donde las consideraciones } \\
\text { ambientales, sociales y económicas estén equilibradas en la búsqueda de una mejor calidad de vida. } \\
\text { Este se consolida a través del conocimiento de los impactos ambientales, implícitos en las problemáticas } \\
\text { que se presentan en las IES; esto conlleva a la puesta en acción de políticas favorables para el medio } \\
\text { ambiente como se mencionaban anteriormente. }\end{array}$ \\
\hline $\begin{array}{l}\text { Familia de información } \\
\text { 4. Estrategias en las IES para la } \\
\text { implementación de la gestión } \\
\text { ambiental }\end{array}$ & $\begin{array}{l}\text { Una de las estrategias que se han utilizado para generar sensibilización e implementar los aspectos } \\
\text { ambientales, consiste en brindar capacitaciones a través de estrategias lúdicas y didácticas que permitan } \\
\text { a las personas tener una cultura más comprometida con el cuidado y buena disposición de los recursos. }\end{array}$ \\
\hline \multirow{3}{*}{$\begin{array}{l}\text { Familia de información } \\
\text { 5. Cultura organizacional }\end{array}$} & $\begin{array}{l}\text { En esta familia aparecen aspectos relacionados con el talento humano en cuanto a sus responsabilidades } \\
\text { y funciones encaminadas a la gestión ambiental, al manual de funciones para el SIG y a la metodología } \\
\text { necesaria para el éxito de las acciones ambientales. }\end{array}$ \\
\hline & $\begin{array}{l}\text { Frente al tema ambiental los resultados señalan la necesidad de planificar acciones con el fin de } \\
\text { involucrar y comprometer a todas las partes interesadas para lograr, desde las actividades propias, el } \\
\text { empoderamiento y la contribución para implementación del SIG. }\end{array}$ \\
\hline & $\begin{array}{l}\text { Los objetivos primordiales son impactar positivamente la cultura organizacional para que todas las partes } \\
\text { interesadas acepten y asuman la política de gestión integral, a la vez que interioricen algunos de los } \\
\text { beneficios que ofrece un SIG implementado. }\end{array}$ \\
\hline $\begin{array}{l}\text { Familia de información } \\
\text { 6. Percepción de la norma }\end{array}$ & $\begin{array}{l}\text { Los resultados recogen las dificultades descritas o insinuadas en las respuestas de las entrevistas acerca } \\
\text { de la implementación de estrategias dirigidas a un sistema de gestión ambiental; están relacionadas en } \\
\text { su mayoría con la falta de concientización de la comunidad universitaria hacia los temas ambientales; } \\
\text { hay ausencia de una conciencia ambientalmente responsable. De igual manera, temas como la ausencia } \\
\text { de políticas, de responsabilidades y autoridades claramente definidas, recursos limitados y falta de } \\
\text { compromiso, son aspectos que han dificultado el camino hacia la consolidación de un sistema de gestión } \\
\text { ambiental. }\end{array}$ \\
\hline
\end{tabular}

Fuente: elaboración propia

Lineamientos para el sistema de gestión integrado bajo la norma NTC-ISO 3001:2015 y NTC-ISO 14001:2015 en instituciones de educación superior

Esta propuesta pretende ser una herramienta que oriente y facilite a las IES tener un sistema integrado de gestión bajo los lineamientos de la norma NTCISO 9001:2015 y NTC-ISO 14001:2015.

Las IES del estudio están certificadas en ISO 9001:2008, por lo que la renovación de la certificación debe hacerse de acuerdo con la versión 2015 y para afrontar la certificación ambiental igualmente deben acogerse a la versión NTC-ISO 14001:2015. Esta propuesta muestra una manera de contar con un SIG incorporado al sistema de gestión propio de cada institución universitaria. Además, los lineamientos están construidos de acuerdo con la nueva estructura de las normas ISO, conocida como HLS o Estructura de Alto Nivel por sus siglas en inglés. 
La nueva estructura introduce unos capítulos, como el análisis del contexto, el pensamiento basado en riesgos y los apoyos o soportes. Desde las miradas y experiencias de las IES, el análisis del contexto aporta oportunidades excepcionales para esa apertura de las organizaciones fuera de sus propios muros, concretando opciones que podrían vehiculizar la realización de aspectos misionales.

En cuanto al pensamiento basado en riesgos, tema fuerte en la HLS, las normas obligan a las organizaciones a prever sus riesgos causados por la incertidumbre y a descubrir sus oportunidades en la línea de la globalización y sus desagregados. Este pensamiento se traduce mediante la prevención de resultados indeseados, oportunidades para alcanzar resultados superiores en los sistemas de gestión.

Es igualmente necesario resaltar el capítulo sobre apoyos que a veces no responden a las exigencias, necesidades y expectativas del SIG. Se trata de asignar en el presupuesto adecuados recursos humanos, financieros, tecnológicos y de tiempo, y, sobre todo, entregarlos todos y oportunamente. Las figuras 1, 2 y 3 muestran una breve descripción de los lineamientos propuestos:

Figura 1. Lineamientos propuestos para un SIG en IES

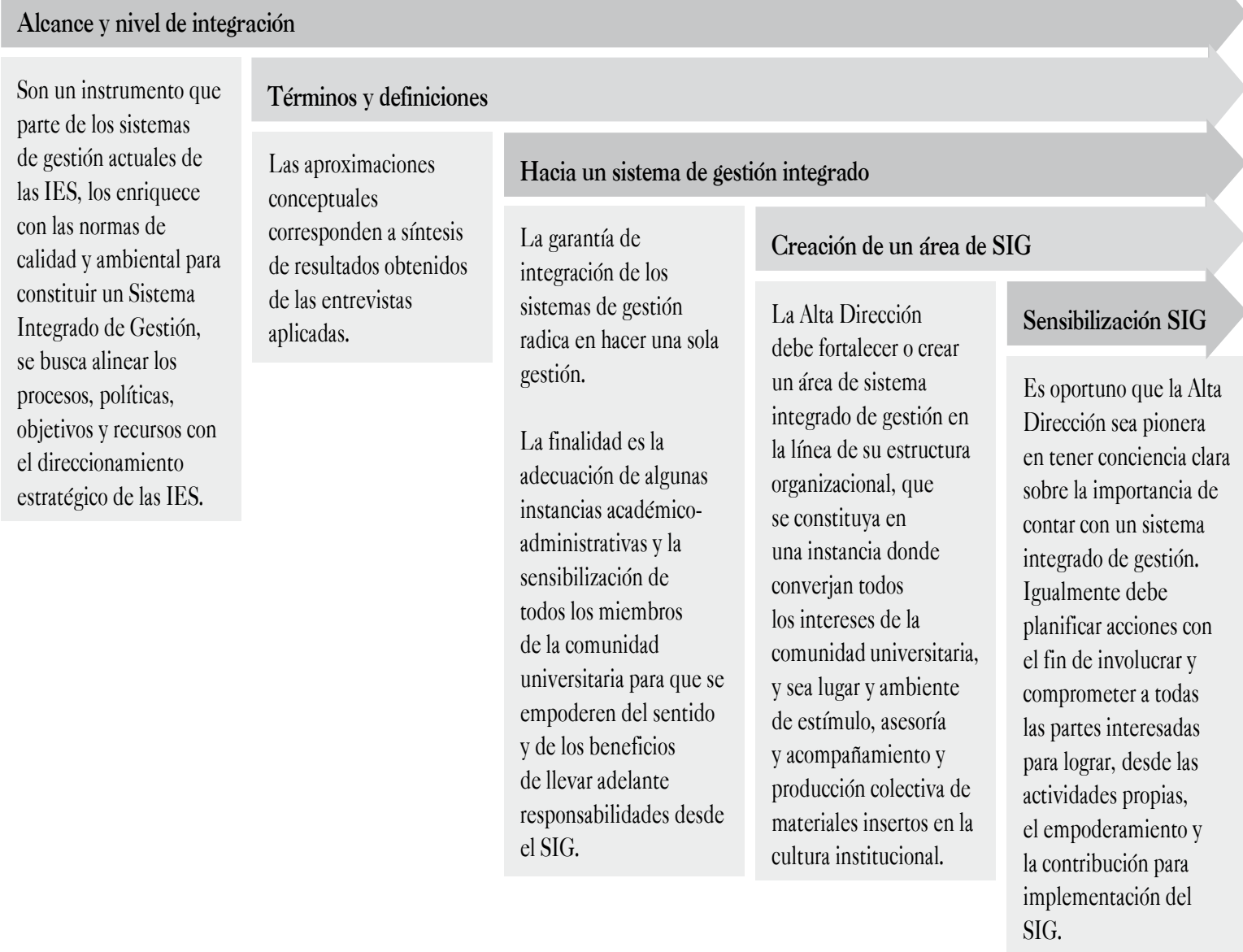

Fuente: elaboración propia 
Figura 2. Lineamientos propuestos para un SIG en IES

Hacia una política de gestion
Alta Dirección debe
integrar y alinear,
desde su planificación
estratégica la definición
de una política de
gestión integral, así
como los objetivos y
las metas que permitan
cumplir con dicha
políitica, ya que al incluir
los temas ambientales
y de calidad en el
contexto institucional se
logra crear una cultura
organizacional en todos
los miembros de la
comunidad universitaria.

Fuente: elaboración propia
Incorporar los requerimientos de los sistemas de gestión estandarizados en el sistema de gestión de la organización
La tarea que se acomete en seguida es la parte central de la propuesta: las IES desarrollan su sistema de gestión y es ahí donde se deben incorporar las normas ISO 9001 y 14001, en su versión 2015.

\section{Figura 3. Lineamientos propuestos para un SIG en IES}

\section{Comprensión de la organización y su contexto}

La Alta Dirección debe llevar a cabo un análisis integral pertinente del contexto, determinar los aspectos externos e internos que afectan la capacidad para logar los resultados del sistema integrado de gestión.

\section{Comprensión expectativas partes interesadas}

Como actividad prioritaria, las IES deben determinar quiénes son sus partes interesadas y disponer de una descripción sobre sus características que puedan ser útiles para que las IES mejoren su desempeño.
Liderazgo y compromiso

La Alta Dirección debe comprender y aceptar que la responsabilidad fundamental y continua sobre el Sistema de gestión y el SIG recae en ella, y que las delegaciones en otras instancias y funcionarios no sustituyen su tarea.

\section{Roles, autoridad y responsabilidad}

La Alta Dirección debe establecer en el Manual de funciones las responsabilidades y competencias necesarias para el mantenimiento y mejora continua del sistema integrado.

\section{Planificación}

Acciones para abordar los riesgos y oportunidades.

Planificación del cambio.

Apoyos
Recursos:
Definir los recursos
necesarios de acuerdo
con las capacidades y
limitaciones internas de
cada IES.
Destinar en el
presupuesto anual
un porcentaje para
la implementación y
mantenimiento del
sistema integrado de
gestión.

Derim fuente para a y la Seguimiento al desarrollo de los servicios prestados.

Definir y documentar los requisitos de los servicios académicos, de investigación y extensión. Acciones de creación y modificación de programas académicos y los impactos ambientales de cada uno e ellos. rencion

\section{Evaluación del desempeño y mejora}

Monitoreo permanente al cronograma de implementación. Auditorias combinadas. 


\section{CONCLUSIONES}

Ninguna de las misiones de las IES de la muestra contiene el compromiso con los sistemas de calidad y ambiental, es decir, esos sistemas no conforman el núcleo misional de las IES, hecho que no favorece o no facilita la implementación del sistema integrado de gestión.

Para las IES del estudio no es claro que implementar un sistema de gestión ambiental contribuye a los fines misionales institucionales. Esto puede explicarse porque no han hecho conciencia generalizada de lo que puede aportar todo lo referente al medio ambiente a la concreción de la formación integral en su desagregado de profesionales íntegros, con una visión sistémica de la vida y de su ejercicio familiar, profesional y ciudadano.

En las IES no se perciben objetivos estratégicos dirigidos a lograr un mejor aprovechamiento de sus recursos, a fin de que sean instituciones ambientalmente sostenibles, preocupadas por mitigar los impactos generados producto de sus actividades y por generar políticas institucionales que sean un modelo a seguir por otras universidades.

En términos generales, para diseñar un sistema de gestión ambiental todas las IES deben identificar claramente sus procesos, y como siguiente paso, realizar una revisión ambiental inicial (RAI) o diagnóstico ambiental a través de la aplicación de listas de chequeo, observación, revisión documental, entrevista u otros instrumentos.

Posteriormente las IES deben adoptar un compromiso ambiental, el cual se estipula en la política ambiental con el fin de dar un horizonte a la gestión. Tal compromiso debe ser acorde con la naturaleza de la IES, convirtiéndose en la base del sistema de gestión ambiental. De esta política surgen los objetivos ambientales, los cuales se estructuran con metas e indicadores.
Para que el sistema de gestión ambiental sea un componente importante dentro de una IES, se debe tener en cuenta su contexto interno y externo, lo cual es relevante en el desarrollo de sus actividades para obtener los resultados previstos que puedan afectar positiva 0 negativamente a la organización o al contexto. Este análisis se debe realizar periódicamente y evidenciar los posibles cambios que se generen.

Un sistema de gestión ambiental para que sea consistente, efectivo e integral debe cumplir con la legislación ambiental vigente, las normas y procedimientos que estén asociados para mitigación o controlar cualquier impacto ambiental y demás instrumentos requeridos para asegurar el cumplimiento de la política y los objetivos y metas ambientales propuestos.

Los futuros investigadores podrín aplicar los lineamientos para sistema de gestión integrado bajo la norma NTC-ISO 9001:2015 e ISO 14001:2015 en las diferentes IES para determinar la eficacia y eficiencia de los mismos.

De igual manera podrín realizar una prueba piloto con los lineamientos establecidos en esta investigación, con el fin de contar con otros criterios diferentes al de la validación por parte de la técnica de jueces.

Otros estudios podrían mirar el nivel de integración de las IES, ya que esta investigación tiene un alcance limitado, debido a que solo se tuvo en cuenta la percepción del personal entrevistado para el diagnóstico del personal.

Se podrán generar proyectos que promuevan sistemas integrados en IES y tengan como referencia todo lo abordado en esta investigación.

Estos lineamientos se pueden aplicar en universidades públicas para probar un ámbito de extensión y ampliación de la utilidad de la herramienta. 


\section{REFERENCIAS}

Bernardo, M., Casadesus, M., Karapetrovic, S., y Heras, I. (2009). La integración de sistemas de gestión basados en estándares internacionales: resultados de un estudio empírico realizado en la CAPV. Journal of Cleaner Producción, 17, 742-750.

Casadesus, M., Karapetrovic, S., y Heras I. (2009). Sistemas de gestión estandarizados: ¿existen sinergias? Revista Europea de Dirección y Economía de la Empresa, 18(2), 161-174.

Cidad, E. (2004). La gestión de la calidad en las organizaciones de educación superior. Aportación del enfoque de la Organización Internacional de Normalización (ISO). Revista Complutense de Educación, 15(2), 647-686.

Ferguson, A., y García, B. B. (2002). Modelos de implantación de los sistemas integrados de gestión de la calidad, el medio ambiente y la seguridad. Investigaciones Europeas, 8(1), 97-118.

Hernández, R., Fernández, C., y Baptista, P. (2014). Fundamentos de metodología de la investigación. México: McGraw-Hill, Interamericana.

Instituto Colombiano de Normas Técnicas y Certificación - Icontec. (2015). Norma Técnica Colombiana ISO 9001, Sistemas de Gestión de la Calidad. Requisitos. Bogotá: Icontec.
Instituto Colombiano de Normas Técnicas y Certificación - Icontec. (2015). Norma Técnica Colombiana ISO 14001, Sistemas de Gestión Ambiental. Requisitos. Bogotá: Icontec.

Jonker, J., \& Karapetrovic, S. (2004). Systems thinking for the integration of management systems. Business Process Management Journal, 10(6), 608615.

Karapetrovic, S. (2003). Musings on integrated management systems. Measuring Business Excellence, 7(1), 4-13.

Karapetrovic, S., \& Willborn, W. (1998). Integration of quality and environmental management systems. The TQM Magasine, 10(3), 204-213.

Ramírez, M., y Manrique, O. (2012). Evidencia de los elementos de la responsabilidad social empresarial en los modelos de la gerencia. Tesis de maestría. Bogotá: Universidad EAN.

G. Wilkinson, B.G. Dale, (1999) "Integrated management systems: an examination of the concept and theory", The TQM Magazine, Vol. 11 Issue: 2, pp. $95-104$.

Rivas, M. I. (2011). Modelo de sistema de gestión ambiental para formar universidades ambientalmente sostenibles en Colombia. Revista Gestión y Ambiente, 4(1), 151-162. 